\title{
Robust topology optimization of photonic crystal waveguides with tailored dispersion properties
}

\author{
Wang, Fengwen; Jensen, Jakob Søndergaard; Sigmund, Ole
}

Published in:

Optical Society of America. Journal B: Optical Physics

Link to article, DOI:

10.1364/JOSAB.28.000387

Publication date:

2011

Document Version

Publisher's PDF, also known as Version of record

Link back to DTU Orbit

Citation (APA):

Wang, F., Jensen, J. S., \& Sigmund, O. (2011). Robust topology optimization of photonic crystal waveguides with tailored dispersion properties. Optical Society of America. Journal B: Optical Physics, 28(3), 387-397. https://doi.org/10.1364/JOSAB.28.000387

\section{General rights}

Copyright and moral rights for the publications made accessible in the public portal are retained by the authors and/or other copyright owners and it is a condition of accessing publications that users recognise and abide by the legal requirements associated with these rights.

- Users may download and print one copy of any publication from the public portal for the purpose of private study or research.

- You may not further distribute the material or use it for any profit-making activity or commercial gain

- You may freely distribute the URL identifying the publication in the public portal 


\title{
Robust topology optimization of photonic crystal waveguides with tailored dispersion properties
}

\author{
Fengwen Wang, * Jakob S. Jensen, and Ole Sigmund \\ Department of Mechanical Engineering, Technical University of Denmark, Nils Koppels Allé, \\ Building 404, 2800 Kgs. Lyngby, Denmark \\ ${ }^{*}$ Corresponding author: fwan@mek.dtu.dk
}

Received August 16, 2010; revised December 9, 2010; accepted December 12, 2010; posted December 15, 2010 (Doc. ID 133488); published February 8, 2011

\begin{abstract}
A robust topology optimization method is formulated to tailor dispersion properties of photonic crystal waveguides, with consideration of manufacturing uncertainties. Slightly dilated and eroded realizations are considered as well as the real structure, and by worst-case optimization, we also ensure a satisfactory performance in the case of an under- or overetching scenario in the manufacturing process. Two photonic crystal waveguides facilitating slow light with group indexes of $n_{g}=25$ and $n_{g}=100$ and bandwidths of $\Delta \omega / \omega=2.3 \%$ and $0.3 \%$, respectively, are obtained through the proposed robust design procedure. In addition, a novel waveguide design with two different constant group index waveguide regions is demonstrated. The numerical examples illustrate the efficiency of the robust optimization formulation and indicate that the topology optimization procedure can provide a useful tool for designing waveguides that are robust to manufacturing uncertainties such as under or overetching. (c) 2011 Optical Society of America
\end{abstract}

OCIS codes: $\quad 000.4430,130.5296,230.7400$

\section{INTRODUCTION}

Slow-light waveguides have a great variety of applications, such as for compact optical delay lines, optical buffers, and enhanced light-matter interaction [1]. Photonic crystal waveguides (PhCWs), generated by missing air holes in a twodimensional (2D) photonic crystal (PhC) slab, facilitate slow-light propagation within the bandgap of the $\mathrm{PhC}$ [2] through strong structural dispersion. The slow-light regime of PhCWs is usually located in the vicinity of the Brillouin zone edge, where the group index diverges to infinity. The slowlight bandwidth becomes narrower as the group index increases and large group-velocity dispersion (GVD) can be observed in the slow-light regime, which can severely distort the optical signal. Therefore, special attention has been paid to these issues [3-7].

The PhCW dispersion properties are strongly sensitive to structural details, and they can be tuned through the design of the supercell, the periodic cell of PhCWs. Previously, it was demonstrated that the dispersion curve can be tailored by different approaches. Slow light with low GVD has been achieved by chirping the waveguide properties [3,4], perturbing the diameters of the air holes [5] or the locations of air holes [6] adjacent to the central defect. However, slow-light propagation is very sensitive to geometrical parameters, and thus very careful experiments are necessary for observation and evaluation of the slow light [7]; manufacturing uncertainties may degrade or destroy the prescribed group velocity. Therefore, a systematic and robust design methodology is highly desirable for PhCW design.

A method that recently has been successfully applied to a range of $\mathrm{PhC}$ based structures and devices is the topology optimization method []. This method, which is based on repeated finite-element analyses and gradient-based optimization updates, was originally developed for mechanical problems, but has more recently been applied to $\mathrm{PhC}$ design in a number of papers [9-11]. A comprehensive review of topology optimization applied to nano-optical design can be found in Ref. [12]. Previously it was demonstrated that topology optimization can be applied to create novel waveguides with enhanced dispersion properties [13]. By maximizing the mode confinement for a prescribed frequency-wavenumber range, Stainko and Sigmund managed to achieve a constant group velocity within a $5 \%$ error interval. The present work addresses the dispersion control problem considered in [13] in a more rigorous manner and simultaneously tackles important manufacturability issues.

A main challenge in topology optimization is the manufacturability of the optimized designs, which includes imposing a minimum length scale on the designs and consideration of manufacturing uncertainties due to under or overetching in the manufacturing process. Different filter schemes have been introduced to control checkerboard instabilities and achieve minimum length scale [14-22]. In order to realize robustness in topology optimization, Sigmund [23] suggested a robust formulation based on dilated, intermediate, and eroded design realizations corresponding to under, normal, and overetching, respectively.

In this work, we focus on robust topology optimization of PhCWs with tailored dispersion properties. Manufacturing uncertainties are approximated by a threshold projection method with different thresholds [24]. Based on Floquet-Bloch type eigenvalue analysis of the supercell, we formulate the objective as the error between the actual group index and a prescribed group index for certain discrete wavenumbers. The robust formulation consists in minimizing the maximum error among the dilated, intermediate and eroded design realizations. Band constraints are introduced to avoid multiple modes and to isolate the designed propagation band. 
This paper is organized as follows. In Section 2 , we present the $2 \mathrm{D}$ model that is used to calculate the band structure of PhCWs and a numerical approximation of the group index. In Section 3, the formulation for robust topology optimization and the associated analytical sensitivities are given. Numerical examples are demonstrated in Section $\underline{4}$ and we summarize in Section $\underline{5}$

\section{PHYSICAL PROBLEM}

PhCWs can facilitate guided modes in the bandgap of PhCs. For a TE-polarized PhCW, the light propagation can be modeled by the Helmholtz equation in the frequency domain

$$
\nabla \cdot\left(\frac{1}{\varepsilon_{r}} \nabla h\right)+\left(\frac{\omega}{c}\right)^{2} h=0,
$$

where $\varepsilon_{r}$ is the space-dependent relative permittivity, $h$ is the magnetic field, $\omega$ is the wave frequency, and $c$ is the speed of light in vacuum.

In this study, we focus on the triangular lattice PhCW based on a silicon membrane $\left(n_{\mathrm{Si}}=3.476, \varepsilon_{\mathrm{Si}}=n_{\mathrm{Si}}^{2}\right)$. As a starting point for our optimization study, we consider the initial waveguide structure illustrated in Fig. 1(a). The dimension of the supercell in the propagation direction is the lattice constant $a$. The line defect is surrounded by 5.5 layers of air holes and the dimension of the supercell perpendicular to the propagation direction is $b=6 \sqrt{3} a$. The diameter of the air holes is $d=0.6 a$. The calculation of the band structure of PhCWs can be formulated as an eigenvalue problem of the supercell with Floquet-Bloch wave boundary conditions. Based on the supercell in Fig. 1(a), the Floquet-Bloch wave boundary conditions can be stated as

$$
h(x, a)=\exp (i k a) h(x, 0) \quad h(0, y)=h(b, y),
$$

where $k$ is the Bloch-wavenumber in the propagation direction.

The discrete expression of Eq. (1) can be obtained using the finite-element method

$$
\left(\mathbf{K}_{k}-\omega^{2} \mathbf{M}\right) \mathbf{h}=0,
$$

where $\mathbf{K}_{k}$ is the global finite-element matrix stemming from the first term in Eq. (1), $\mathbf{h}$ is a vector of discretized nodal values of magnetic fiel $h$, and $\mathbf{M}$ is the global finite-element matrix from the second term in Eq. (1). The wavenumber dependency of $\mathbf{K}_{k}$ is introduced by the implementation of the boundary conditions in Eq. (2) through the penalty approach.

Figure 1(b) depicts the band structure of the supercell in Fig. 1(a). The gray regions indicate the slab mode region (modes in the crystal not confined to the line defect). The dotted curve denotes the light line $\omega=c k$, which represents the condition for light leaking in the out-of-plane direction. Beneath the light line, the higher index waveguide core pulls down discrete guided modes into the bandgap [2]. Because of the presence of a lateral symmetry, the guided modes can be classified as laterally even modes (solid curve) and laterally odd modes (dashed curve). In this paper, only even modes are considered for guided waves.

Figure $1(\mathrm{~b})$ shows that the even band flattens out as it approaches the band edge and the group velocity of the guided mode decreases to zero. The group velocity $v_{g}$ of a guided mode with frequency $\omega$ is defined as the band slope at frequency $\omega$ :

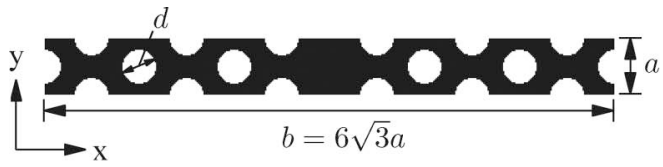

(a)

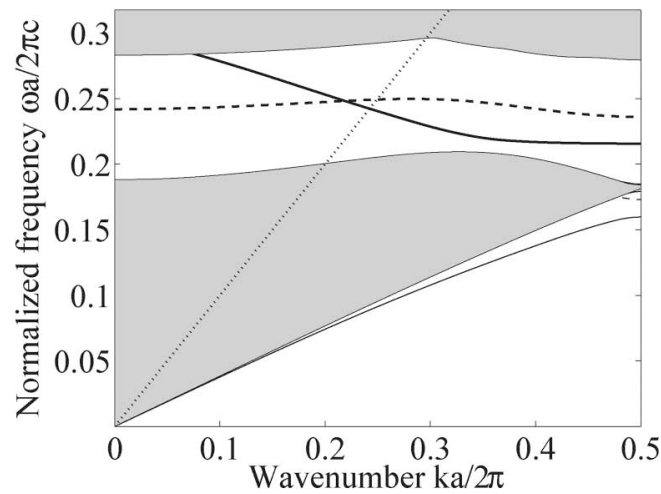

(b)

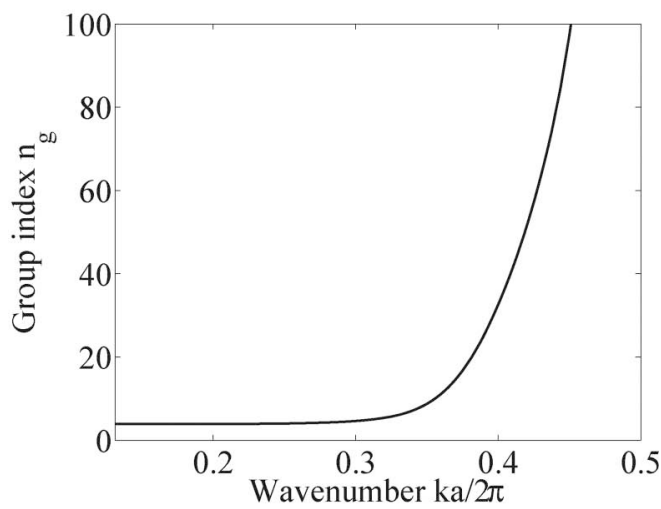

(c)

Fig. 1. (a) Schematic illustration of the supercell of a triangular lattice PhCW. (b) Corresponding band structure. Gray region indicates slab mode region, the dotted curve represents the light line, the solid curves denote the even guided modes, and the dashed curves denote the odd guided modes. (c) Group index of the even guided modes in the bandgap.

$$
v_{g}=\frac{\partial \omega}{\partial k}
$$

The even band has a negative band slope and thus the group index $n_{g}$ for the even guided mode is calculated by

$$
n_{g}=-c \frac{\partial k}{\partial \omega} .
$$

Numerically, we approximate the group index for frequency $\omega_{n}\left(k_{i}\right)$ by

$$
n_{g}\left(\omega_{n}\left(k_{i}\right)\right)=\frac{c\left(k_{i}-k\right)}{\omega_{n}(k)-\omega_{n}\left(k_{i}\right)},
$$

where $n$ is the band number of the designed band in order of increasing frequency and $k$ is a wavenumber close to $k_{i}$. This method is sufficiently accurate if $k$ is sufficiently close to $k_{i}$.

The group index plot [Fig. 1(c)] shows that the mode is index- guided in the fast light regime $\left(n_{g} \approx n_{\mathrm{Si}}\right)$, whereas in the slow-light regime $\left(n_{g} \gg 1\right)$, the mode is bandgap guided. The group index diverges near the band edge and the group index here is strongly dependent on the frequency. Thus a 


$$
\begin{aligned}
& \min _{\rho_{j}} \max _{q} \max _{k_{i}} f\left(\bar{\rho}^{q}\right)=\left(\frac{c\left(k_{i}-k_{i-1}\right)}{\omega_{n}^{q}\left(k_{i-1}\right)-\omega_{n}^{q}\left(k_{i}\right)}-n_{g}^{*}\right)^{2} \quad \text { s.t. } \quad\left[\mathbf{K}_{k}^{q}-\left(\omega^{q}\right)^{2} \mathbf{M}^{q}\right] \mathbf{h}^{q}=0 \quad \max _{k_{i i}} \omega_{n-1}^{q}\left(k_{i i}\right) \leq a_{1} \min _{k_{i}} \omega_{n}^{q}\left(k_{i}\right) \\
& \omega_{n}^{q}(0) \geq a_{2} \max _{k_{i}} \omega_{n}^{q}\left(k_{i}\right) \quad \min _{k_{i i}} \omega_{n+1}^{q}\left(k_{i i}\right) \geq a_{2} \max _{k_{i}} \omega_{n}^{q}\left(k_{i}\right) \quad f_{v}=\frac{\sum_{j} \bar{\rho}_{j}^{d} v_{j}}{\sum_{j} v_{j}} \leq f_{v}^{*} \\
& 0 \leq \rho_{j} \leq 1 \quad j=1, \ldots, N, \quad i=2, \ldots, m, \quad a_{1}<1, \quad a_{2}>1, \quad q=\{d, i, e\}
\end{aligned}
$$

large GVD is obtained ( $\left.\mathrm{GVD}=c^{-1} \mathrm{~d} n_{g} / \mathrm{d} \omega\right)$, which distorts the waveform of optical signals and, moreover, the bandwidth becomes narrower for the slow light.

\section{ROBUST TOPOLOGY OPTIMIZATION PROBLEM}

\section{A. Formulation of Robust Design}

In many applications, it is highly desirable to reduce the GVD and extend the bandwidth for slow-light operation. This can be realized through a proper modification of the waveguide geometry. In topology optimization, the elementwise design variables $\rho$ are introduced to represent the material distribution in the supercell. These design variables are used to control the dielectric permittivity in each element. The optimization problem can be formulated as minimizing the error between the actual group index and the prescribed group index for certain discrete wavenumbers $k_{i}$, in order to reduce the GVD and extend the bandwidth of the slow light (shown in Fig. 2). In order to realize the desired design robustness, the underetched structure (dilated design, $\bar{\rho}^{d}$ ), normal etched structure (intermediate design, $\bar{\rho}^{i}$ ) and overetched structure (eroded design, $\bar{\rho}^{e}$ ) in the manufacturing process can be considered with a min-max optimization formulation [23]. Robust topology optimization for tailoring dispersion properties is achieved by minimizing the maximum error for certain discrete wavenumbers for the three design realizations.

In order to avoid multimode interference for the tailored modes, band constraints are implemented. First, the lower and upper bands should be kept away from the design (slow light) band. Also, as Fig. 1(b) shows, there is interaction between guided odd and even bands. In order to keep the odd band away from the designed even modes, an additional constraint is implemented on the odd band for $k_{i}=0$ (shown in Fig. 2). The robust formulation can be stated as

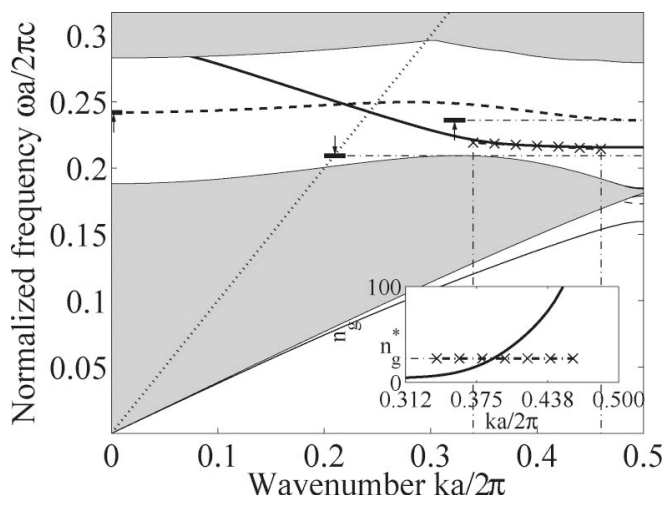

Fig. 2. Schematic illustration of objective and constraints in the robust formulation. The crosses denote the prescribed properties, upward arrow indicates pushing upward, downward arrow indicates pushing downward, and the inset shows the prescribed group index and actual group index versus wavenumber. where $\rho_{j}$ is the design variable for element $j$, The superscript $q$ denotes the design type among the three different design realizations; $q=\{d, i, e\}$ indicates the dilated, intermediate, and eroded designs, respectively; $\bar{\rho}^{q}$ is the physical density vector of the structure $q ; k_{i}$ is the discrete design wavenumber; $\omega_{n}^{q}\left(k_{i}\right)$ is the $n$th order frequency corresponding to wavenumber $k_{i}$ for the design $q ; n_{g}^{*}$ is the prescribed group index; $k_{i i}$ is the discrete wavenumber for lower band $\left(\omega_{n-1}^{q}\left(k_{i i}\right)\right)$ and upper band $\left(\omega_{n+1}^{q}\left(k_{i i}\right)\right) ; a_{1}$ and $\alpha_{2}$ represent the band constraints; $N$ is the total element number; $v_{j}$ is the volume of element $j ; f_{v}$ is the actual volume fraction of the total material, $f_{v}^{*}$ is the given volume fraction; and $m$ is the total number of the design wavenumbers $k_{i}$. The volume constraint is implemented on the dilated design realization.

\section{B. Numerical Approximation of Manufacturing Uncertainties}

The basis for the approximation of under, normal, and overetching in the manufacturing process is the traditional density filter introduced by Bruns and Tortorelli [16] and Bourdin [17]

$$
\tilde{\rho}_{e}=\frac{\sum_{j \in N_{e}} w\left(\mathbf{x}_{j}\right) v_{j} \rho_{j}}{\sum_{j \in N_{e}} w\left(\mathbf{x}_{j}\right) v_{j}}
$$

where $\tilde{\rho}_{e}$ is the filtered density of element $e, \mathbf{x}_{j}$ is the location of element $j, N_{e}$ is the neighborhood of element $e$ within a certain filter radius $r$ specified by

$$
N_{e}=\left\{j \mid\left\|\mathbf{x}_{j}-\mathbf{x}_{e}\right\| \leq r\right\},
$$

and $w\left(\mathbf{x}_{j}\right)$ is the weighting factor defined as $w\left(\mathbf{x}_{j}\right)=$ $r-\left\|\mathbf{x}_{j}-\mathbf{x}_{e}\right\|$.

Based on the filtered density, the under, normal, and overetching can be realized based on a threshold projection by choosing different thresholds. For a given threshold $\eta$, the

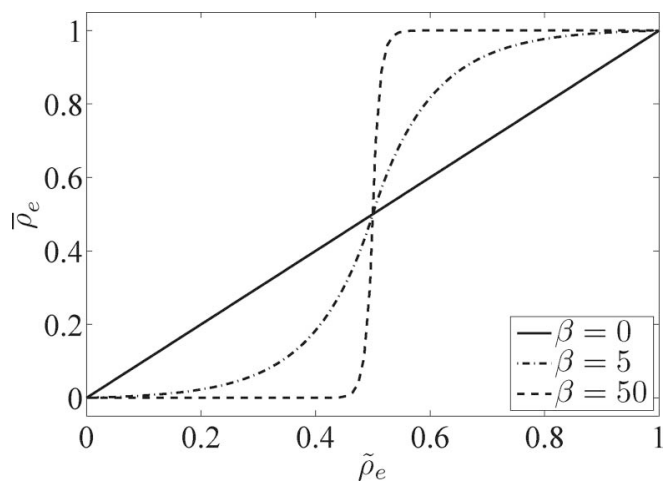

Fig. 3. Illustration of the smoothed threshold projection for $\eta=0.5$ and different values of $\beta$. 


\section{Design domain}

Fig. 4. Illustration of design domain. The dash-dot curves denote the symmetric axes of design domain.

physical density $\bar{\rho}_{e}$ can be approximated by a smooth function governed by the parameter $\beta$ (shown in Fig. $\underline{3}$ for $\eta=0.5$ ):

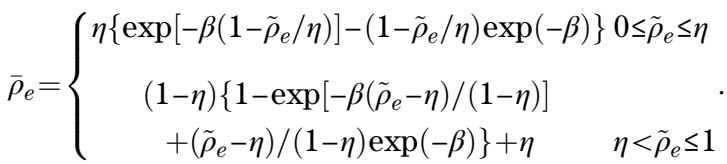

Three threshold values are chosen as $\eta_{d}, \eta_{i}$, and $\eta_{e}$ corresponding to the dilated, intermediate, and eroded design. They satisfy $0 \leq \eta_{d}<\eta_{i}<\eta_{e} \leq 1$. By controlling the threshold values, we control the manufacturing tolerance between the dilated, intermediate, and eroded designs. More details can be found in [24].

\section{Sensitivity Analysis}

The interpolation of the relative permittivity of element $e$ in the design domain is given by a linear function based on the inverse permittivity

$$
\frac{1}{\varepsilon_{e}^{q}}=\left(1-\bar{\rho}_{e}^{q}\right) \frac{1}{\varepsilon_{1}}+\bar{\rho}_{e}^{q} \frac{1}{\varepsilon_{2}},
$$

where $\varepsilon_{1}=1$ is the relative permittivity of air and $\varepsilon_{2}=\varepsilon_{\mathrm{Si}}$ is the relative permittivity of silicon. The sensitivity of the objective and constraints can be calculated separately for each structure by the standard chain rule [20]

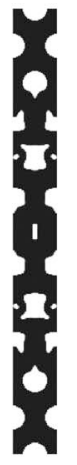

(a)

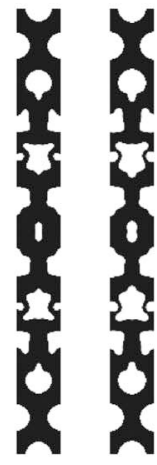

(b)

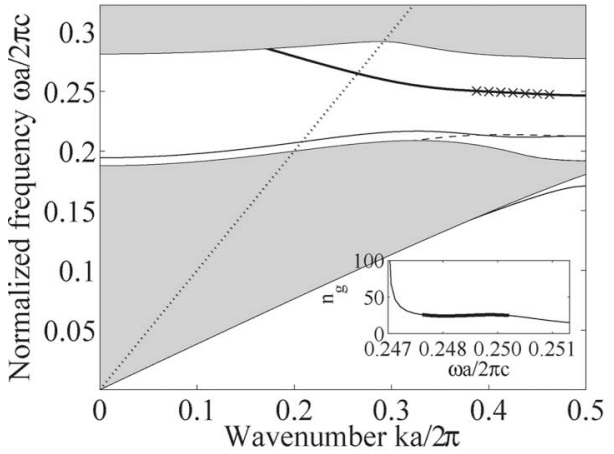

(e)

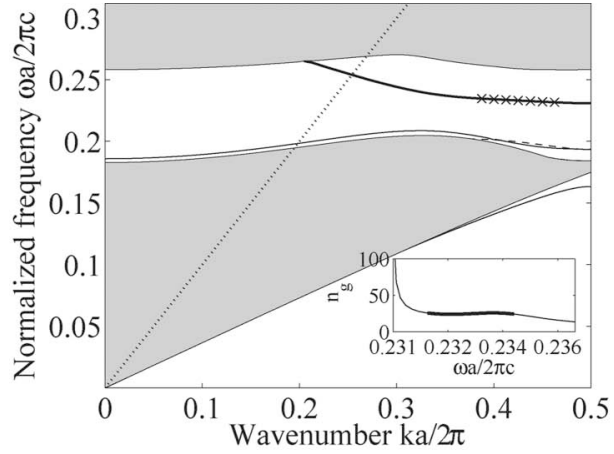

(d)

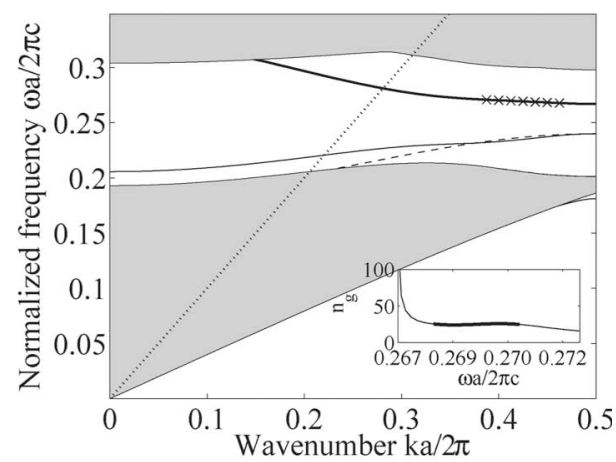

(f)

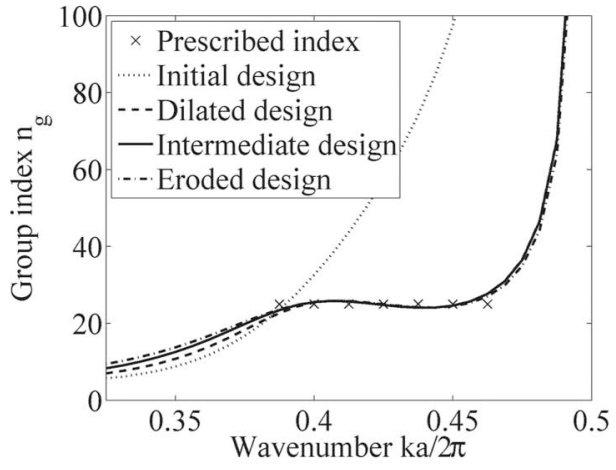

(g)

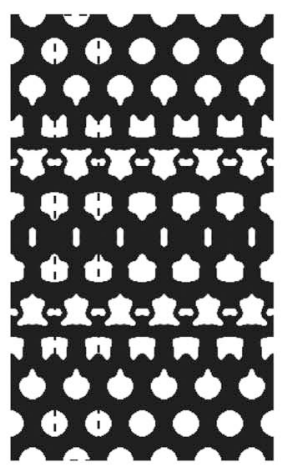

(h)

Fig. 5. Robust design of PhCWs. (a) Dilated design. (b) Intermediate design. (c) Eroded design. (d) Band structure of dilated design. The crosses indicate the design range and the bold curve in the inset denotes the bandwidth of prescribed group index. (e) Band structure of intermediate design. (f) Band structure of eroded design. (g) Group index of the different design realizations and prescribed group index. (h) PhCW constituted by intermediate design. 


$$
\frac{\partial F}{\partial \rho_{j}}=\sum_{e \in N_{j}} \frac{\partial F}{\partial \bar{\rho}_{e}^{q}} \frac{\partial \bar{\rho}_{e}^{q}}{\partial \tilde{\rho}_{e}} \frac{\partial \tilde{\rho}_{e}}{\partial \rho_{j}}
$$

where $F$ denotes the objective $f$ or constraints.

The sensitivity of the objective with respect to the physical density can be expressed as

$$
\begin{aligned}
\frac{\partial f\left(\bar{\rho}^{q}\right)}{\partial \bar{\rho}_{e}^{q}}= & -2\left(\frac{c\left(k_{i}-k_{i-1}\right)}{\omega_{n}^{q}\left(k_{i-1}\right)-\omega_{n}^{q}\left(k_{i}\right)}-n_{g}^{*}\right) \\
& \times \frac{c\left(k_{i}-k_{i-1}\right)}{\left(\omega_{n}^{q}\left(k_{i-1}\right)-\omega_{n}^{q}\left(k_{i}\right)\right)^{2}}\left(\frac{\partial \omega_{n}^{q}\left(k_{i-1}\right)}{\partial \bar{\rho}_{e}^{q}}-\frac{\partial \omega_{n}^{q}\left(k_{i}\right)}{\partial \bar{\rho}_{e}^{q}}\right) .
\end{aligned}
$$

The sensitivity of the eigenvalues and also for multiple eigenvalues can be found in many papers, e.g., Seyranian et al. [25] and Pedersen and Nielsen [26]. Based on Eq. (므), the derivative of the physical density $\bar{\rho}_{e}^{q}$ with respect to the filtered density $\tilde{\rho}_{e}$ is

$$
\frac{\partial \bar{\rho}_{e}^{q}}{\partial \tilde{\rho}_{e}}=\left\{\begin{array}{ll}
\beta \exp \left[-\beta\left(1-\tilde{\rho}_{e} / \eta_{q}\right)\right]+\exp (-\beta) & 0 \leq \tilde{\rho}_{e} \leq \eta_{q} \\
\beta \exp \left[-\beta\left(\tilde{\rho}_{e}-\eta_{q}\right) /\left(1-\eta_{q}\right)\right]+\exp (-\beta) & \eta_{q}<\tilde{\rho}_{e} \leq 1
\end{array} .\right.
$$

The sensitivity of the filtered density $\tilde{\rho}_{e}$ with respect to the design variable $\rho_{j}$ can be calculated by

$$
\frac{\partial \tilde{\rho}_{e}}{\partial \rho_{j}}=\frac{w\left(\mathbf{x}_{j}\right) v_{j}}{\sum_{i \in N_{e}} w\left(\mathbf{x}_{i}\right) v_{i}} .
$$

\section{Numerical Implementation}

Since the supercell is the periodic cell of PhCWs, the neighborhood of element $e, N_{e}$ includes the neighbor elements in the previous or next supercell in the density filter. Furthermore, in order to keep the smoothness between design domain and nondesign domain, the physical densities of the elements adjacent to the design domain are updated according to the design variables. The optimization problem is solved by the globally convergent version of the method of moving asymptotes (GCMMA) from Svanberg [27]. The full optimization procedure is as follows:

1. Set the design domain and choose the $\eta$ set: $\eta_{d}, \eta_{i}$, and $\eta_{e}$.

2. Build the neighborhood $\mathbf{N}$ for changeable elements.

3. Initialize design variables $\rho$.

4. Compute the dilated $\left(\bar{\rho}^{d}\right)$, intermediate $\left(\bar{\rho}^{i}\right)$, and eroded $\left(\bar{\rho}^{e}\right)$ design realizations based on the design variables.

5. Solve the eigenvalue problems based on the dilated, intermediate, and eroded designs.

6. Calculate objectives and constraints and corresponding sensitivities.

7. Update design variables $\rho$ using GCMMA.

8. Calculate max design variable change $\Delta \rho$ and max objective change $\Delta f$.
9. For every fortieth iteration or if $(\{\Delta \rho<1 e-3$ or $\Delta f<$ $1 e-3\}$ and $\left.\beta<\beta_{\max }\right)$, set $\beta=1.3 \beta$.

10. If $(\Delta \rho<1 e-4$ or $\Delta f<1 e-4)$ and $\beta \geq \beta_{\max }$, terminate, else, goto 4 .

Here the maximum value of $\beta$ is $\beta_{\max }=50$.

The optimization procedure is implemented in MATLAB and runs in parallel on a standard four-processor PC. The optimization converges in 400-500 GCMMA iterations, for which each GCMMA iteration includes three or four inner-iterations and takes 3-4 min for seven discrete wavenumbers.

\section{RESULTS}

It was shown in [5] that the first two rows of air holes adjacent to the waveguide core have a significant influence on the group index. Here we add one and one-quarter rows and set the first three and one-quarter rows adjacent to the waveguide core as the design domain. A symmetry condition is introduced to ensure symmetric designs (shown in Fig. 4). The initial design is illustrated in Fig. 1(a). The supercell is discretized with $40 \times 408$ quadrilateral four-node elements. The volume constraint is $f_{v}^{*}=0.8$, the filter radius $r$ is set as $1 / 8 a$, and the threshold set is $\eta=\{0.35,0.5,0.65\}$. In order to measure the separation between the designed band and the other bands, we define the relative band distance: the relative band distance is defined as the band distance to mid-distance frequency ratio $\Delta \omega / \omega_{m}, \Delta \omega$ is the minimum of the minimal distance between upper band and lower band, and the distance between the minimal odd mode and lower band and $\omega_{m}$ is the frequency at the middle of the band distance.

\section{A. Robust Design of PhWGs}

In the first example, we try to design PhCWs with the constant group index of $n_{g}=25$ in the wavenumber range $k \in[0.3875,0.4625] 2 \pi / a$. The target wavenumbers $k_{i}$ are set to seven equidistant points in above wavenumber range. The final robust design is shown in Fig. $\underline{5}$. Figures $\underline{5(\mathrm{a})}-\underline{5(\mathrm{c})}$ display the dilated, intermediate, and eroded design realization. The corresponding band structures [Figs. 5(d)-5(f)] show that the design regions are well isolated from other modes. By studying the band structures and the inset group index plots, we can see that the guided modes with almost constant group index are located at different frequency ranges for the three design realizations. This is an expected feature, since the frequency scales as $1 / \sqrt{\varepsilon}$ in a medium of dielectric constant $\varepsilon$. The volume fraction of silicon decreases from the dilated design to the eroded design; therefore, the average dielectric constant decreases from the dilated design to the eroded design, and correspondingly, the location of the designed band should increase in frequency. Figure 5(g) shows the group index plot of all the designs versus wavenumber $k$. It

\begin{tabular}{|c|c|c|c|c|}
\hline Design & $f_{v}$ & Band distance $(2 \pi c / a)$ & Bandwidth $(2 \pi c / a)$ & Error \\
\hline Dilated & 0.740 & $21.1 \%$ in $[0.20866,0.25802]$ & 0.00250 in $[0.23188,0.23438]$ & $3.6 \%$ \\
\hline Intermediate & 0.678 & $24.6 \%$ in $[0.21670,0.27749]$ & 0.00250 in $[0.24760,0.25011]$ & $3.7 \%$ \\
\hline Eroded & 0.613 & $21.5 \%$ in $[0.24000,0.29783]$ & 0.00250 in $[0.26800,0.27050]$ & $3.3 \%$ \\
\hline
\end{tabular}
can be seen that all the design realizations have equally good performance in the design region. Therefore, almost constant group velocity also is achieved when the design is slightly

Table 1. Performance of Different Designs in Fig. 5 


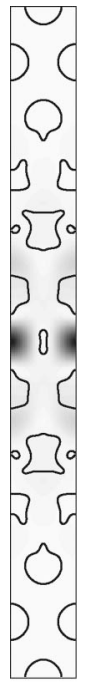

(a) (b)

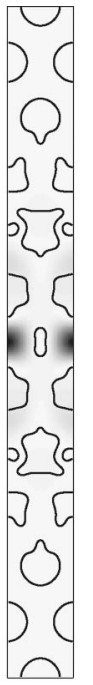

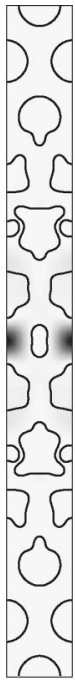

(c)

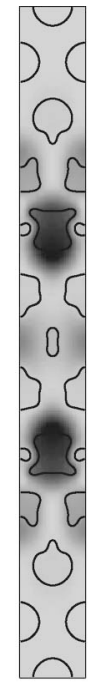

(d)

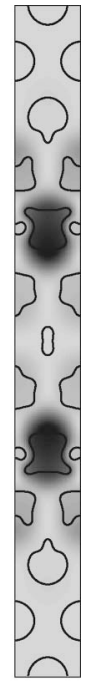

(e)
Fig. 6. Amplitudes of the magnetic field at $k_{i}=0.4252 \pi / a$. (a) Amplitude of the magnetic field for dilated design. (b) Amplitude of the magnetic field for intermediate design. (c) Amplitude of the magnetic field for eroded design. (d) Amplitude of the lower odd magnetic mode for intermediate design. (e) Amplitude of the lower even mode for intermediate design.

under or overetched during the manufacturing process. A constant group velocity is achieved by all three design realizations in the wavenumber range $k \in[0.39375,0.45625] 2 \pi / a$, which is smaller than the target wavenumber range. This is due to the numerical calculation of the group index based on finite differences [Eq. (6)], which causes the errors between actual group index and prescribed group index at the first and last wavenumbers to be much larger than for the other target wavenumbers.

Detailed properties of the three design realizations are shown in Table 1 . Among the three design realizations, the dilated one has the smallest relative band distance. The same bandwidth of $\Delta \omega=0.00252 \pi c / a$ can be achieved by all three of the design realizations in different frequency ranges. The frequency ranges of the bandwidths reveal that the designed modes are located near the center of the corresponding distance. The maximum error of the constant group index is limited to $3.7 \%$.

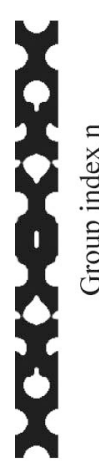

(a)

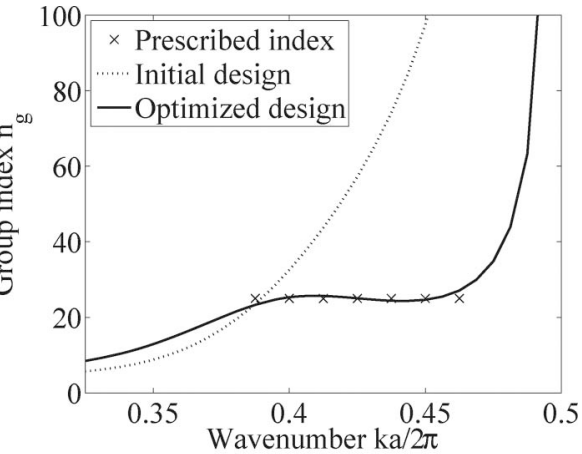

(b)
Fig. 7. Optimized design of $\mathrm{PhCW}$ without considering robustness for $\eta=0.5$. (a) Optimized design. (b) Group index of different designs and prescribed group index.

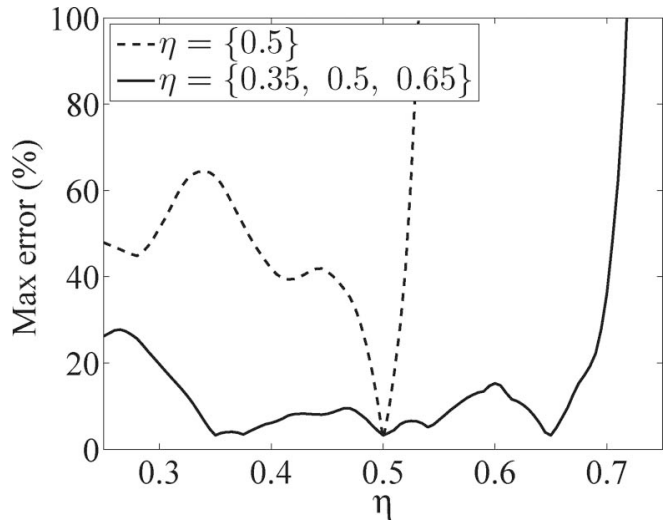

Fig. 8. Maximum error between actual group index and prescribed group index in the design wavenumber range versus $\eta$ for normal and robust formulation.

The band structures of the robust design show that there are two other bands between the slab mode region and the designed band. These two bands interact and degenerate at the band edge. Whereas the designed mode is confined in the waveguide core for all the three design realizations (Fig. 6), the modes of the other two bands are confined in the design domain, but not in the waveguide core.

In order to compare normal nonrobust optimization and robust optimization, we perform a normal optimization for $\eta=$ 0.5 with the same parameter set as in above case. The final design is shown in Fig. 7. Figure 8 depicts the maximum error between actual group index and the prescribed group index versus $\eta$ for the normal design (Fig. 7) and the robust design (Fig. 5). The best performance is obtained by the structures corresponding to the design $\eta, \eta=0.5$ for the normal design and $\eta=\{0.35,0.5,0.65\}$ for the robust design. Compared with the normal optimization, which degenerates for even very small etching errors, the performance of the PhCW has been improved significantly through the robust optimization approach. The robust optimization formulation ensures that the structure realizations corresponding to $\eta \in\left[\eta_{d}, \eta_{e}\right]$ preserve good performance, and the maximum error is limited within $15.3 \%$. An even better performance could be obtained by considering more values of $\eta$ in the interval of $\left[\eta_{d}, \eta_{e}\right]$, but at the expense of increased computation time.

\section{B. Robust Design of PhWGs with Smaller GVD}

In this example, we investigate the influence of the band constraints on the group index and aim to decrease the error of the constant group index. The band constraints are relaxed slightly, i.e., given a smaller relative band distance. The other parameters are kept exactly the same as in the first case. The final design is shown in Fig. 9 illustrating that the topology of the design is quite different in comparison to the first case. There are topological differences between the eroded and the other two design realizations and small isolated components show up in the eroded design realization. All of the conclusions about the frequency shift and confinement drawn from the first case still apply in this case: the frequency of the band shifts upwards from the dilated to the eroded design and the designed modes are confined to the waveguide core [shown in Figs. 9(e)-9(g)]. The group index plot [Fig. 9(i)] shows that a group index of $n_{g}=25$ is achieved with negligible error by all three designs. The smallest bandwidth of $\Delta w=0.00252 \pi c / a$ can be achieved by all the three designs 


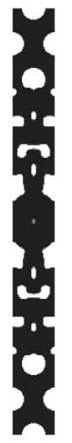

(a)

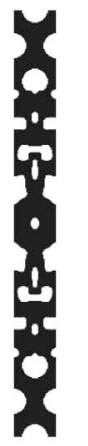

(b)

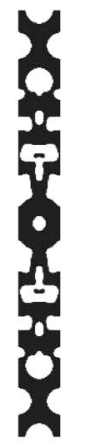

(c)

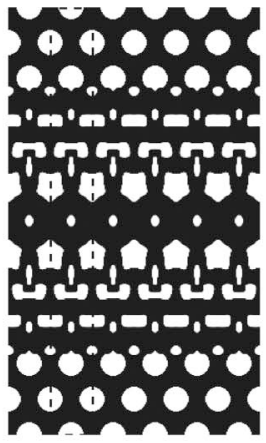

(d)

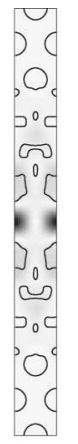

(e)

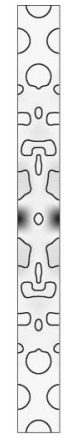

(f)

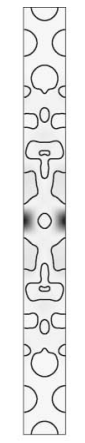

(g)

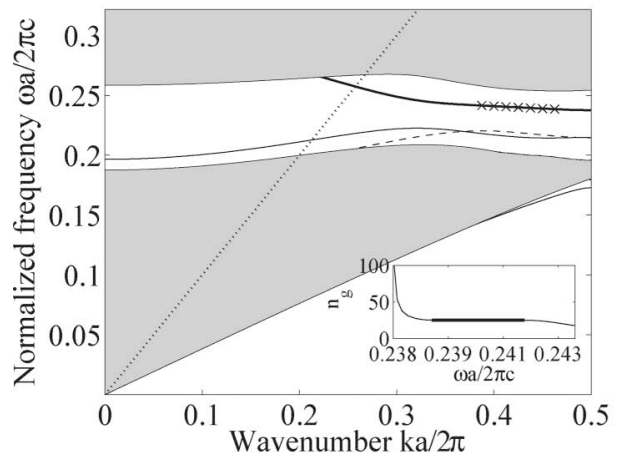

(h)

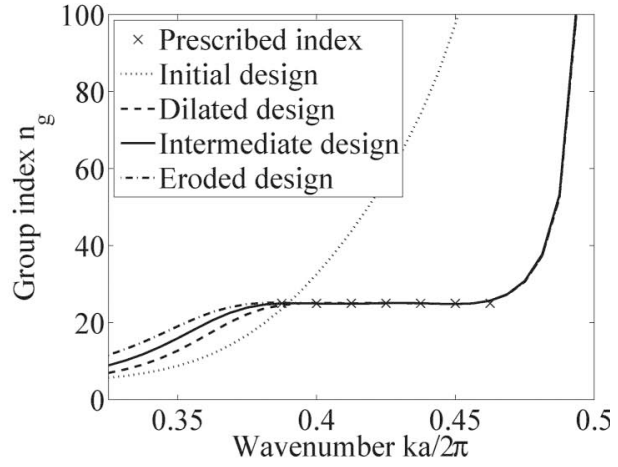

(i)

Fig. 9. Robust design of PhCWs with small GVD. (a) Dilated design. (b) Intermediate design. (c) Eroded design. (d) PhCW constituted by intermediate design. (e) Amplitude of the magnetic field at $k_{i}=0.4252 \pi / a$ for dilated design. (f) Amplitude of the magnetic field at $k_{i}=0.4252 \pi / a$ for intermediate design. (g) Amplitude of the magnetic field at $k_{i}=0.4252 \pi / a$ for eroded design. (h) Band structure of intermediate design. (i) Groups indexes of the different design realizations and prescribed group index.

within an error of $0.8 \%$. Comparison between the first example and this example reveals that the improvement of the dispersion properties can be obtained at the expense of the relative band distance (Table 2).

\section{Extension of the Bandwidth}

Two key parameters of slow light in PhCWs are the dispersion properties and the bandwidth. In the first two examples, we have demonstrated that the GVD of the slow-light regime can be reduced significantly, and a constant group velocity with very small error can be obtained for a certain bandwidth. In this example, we aim to design PhCWs with enlarged bandwidth of the constant group index. To achieve this, we extend the design wavenumber range to $k \in[0.3,0.47] 2 \pi / a$ by setting 10 equidistant target wavenumber $k_{i}$ in above range. The final design is presented in Fig. 10. It is seen that a broader bandwidth is obtained in this case. An almost constant group velocity of $v_{g}=0.04 \mathrm{c}$ can be achieved for the wavenumber range $k \in[0.30625,0.46250] 2 \pi / a$ by all the three design realizations.

Table 3 shows the properties of all of the design realizations. The smallest bandwidth of $\Delta \omega=0.006252 \pi c / a$ can be achieved by all design realizations with the maximum error of $2.6 \%$. Compared to the previous example, the bandwidth is more than doubled, at the expense of a slightly increased error. Examining the guided frequency range and the design wavenumber range, we can see that the largest frequency of the eroded design is close to the light line, which indicates that this bandwidth is close to the largest bandwidth we can obtain.

\section{Design of PhCWs with Different Constant Group Indexes}

In this example, we explore the ability of the robust design formulation further by designing a $\mathrm{PhCW}$, which exhibits two different constant group indexes over different frequency ranges. Our prescribed group indexes are $n_{g}^{*}=20$ for five equidistant design wavenumbers in the wavenumber range of $k \in[0.32,0.37] 2 \pi / a$ and $n_{g}^{*}=25$ for five equidistant design wavenumbers in the wavenumber range of $k \in[0.41,0.46] 2 \pi / a$.

The final design is presented in Fig. 11. The group index plot [Fig. 11(i)] shows two relatively flat parts corresponding to the group indexes of $n_{g}=20$ and $n_{g}=25$. The constant group index of $n_{g}=20$ can be achieved in the wavenumber range of $k \in[0.32500,0.36875] 2 \pi / a$ and the constant group

Table 2. Performance of Different Designs in Fig. 9

\begin{tabular}{lcccc}
\hline Design & $f_{v}$ & Band distance $(2 \pi c / a)$ & Bandwidth $(2 \pi c / a)$ & Error \\
\hline Dilated & 0.761 & $11.2 \%$ in $[0.21129,0.23644]$ & 0.00250 in $[0.22243,0.22493]$ & $0.6 \%$ \\
Intermediate & 0.695 & $13.0 \%$ in $[0.22265,0.25367]$ & 0.00275 in $[0.23881,0.24156]$ & $0.7 \%$ \\
Eroded & 0.617 & $13.2 \%$ in $[0.24338,0.27796]$ & 0.00325 in $[0.26087,0.26412]$ & $0.8 \%$ \\
\hline
\end{tabular}




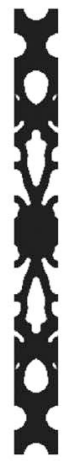

(a)

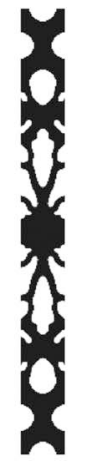

(b)

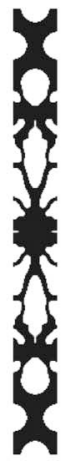

(c)

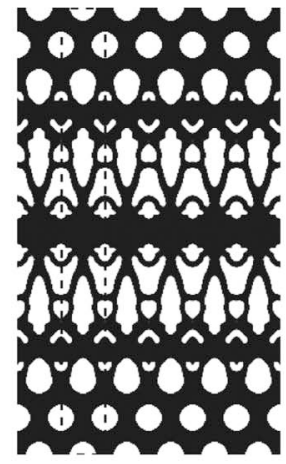

(d)

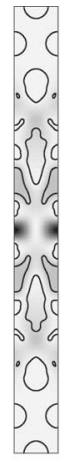

(e)

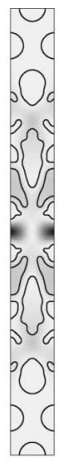

(f)

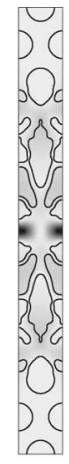

(g)

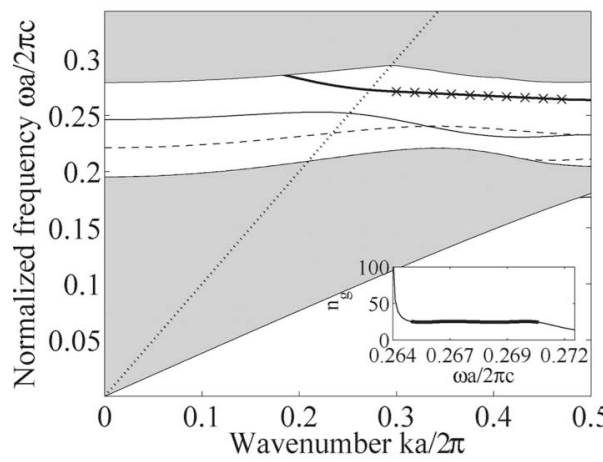

(h)

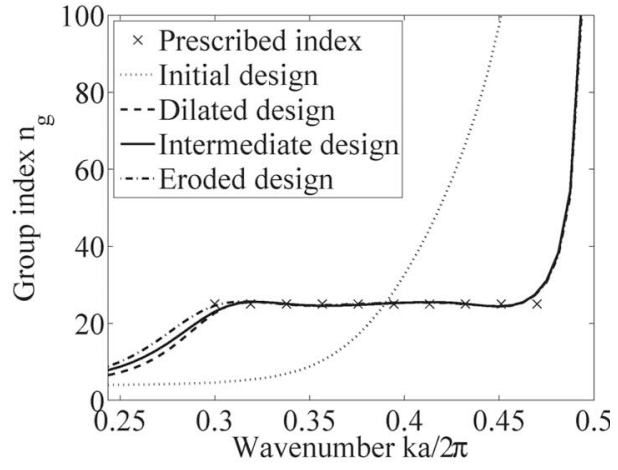

(i)

Fig. 10. Robust design of PhCWs with large bandwidth. (a) Dilated design. (b) Intermediate design. (c) Eroded design. (d) PhCW constituted by intermediate design. (e) Amplitude of the magnetic field at $k_{i}=0.3762 \pi / a$ for dilated design. (f) Amplitude of the magnetic field at $k_{i}=0.3762 \pi / a$ for intermediate design. (g) Amplitude of the magnetic field at $k_{i}=0.3762 \pi / a$ for eroded design. (h) Band structure of intermediate design. (i) Groups indexes of the different design realizations and prescribed group index.

index of $n_{g}=25$ can be achieved in the wavenumber range of $k \in[0.41250,0.45625] 2 \pi / a$ by all the three design realizations.

The detailed properties of these three design realizations are listed in Tables $\underline{4}$ and $\underline{5}$. The bandwidth of $n_{g}=20$ is $\Delta \omega=$ $0.00252 \pi c / a$ with maximum error of $1.7 \%$ and the bandwidth of $n_{g}=25$ is $\Delta \omega=0.001752 \pi c / a$ with maximum error of $1.8 \%$. Among the three design realization, the eroded design exhibits the largest bandwidths for both group indexes.

\section{E. Further Discussion}

The isolated components, which show up in the eroded designs in the second and fourth examples, are not suitable for membrane waveguides. Therefore, further consideration should be given to prevent the isolated components during the optimization process. The isolated components cause the fundamental free mechanical vibration frequency of the supercell to be zero. Therefore, a fundamental mechanical vibration frequency constraint is added to the optimization formulation to prevent isolated components in the designs. The free mechanical vibration problem can be solved as an eigenvalue problem of the supercell under the following boundary conditions:

$$
\mathbf{u}(x, a)=\mathbf{u}(x, 0) \quad \mathbf{u}(0, y)=\mathbf{u}(b, y)=0 .
$$

The mechanical properties are interpolated based on the SIMP model [ㅁ] as

$$
\begin{aligned}
E_{e}^{q} & =E_{\min }+\left(E_{1}-E_{\min }\right)\left(\bar{\rho}_{e}^{q}\right)^{p}, \\
m_{e}^{q} & =m_{\min }+\left(m_{1}-m_{\min }\right) \bar{\rho}_{e}^{q},
\end{aligned}
$$

where $E_{e}^{q}$ and $m_{e}^{q}$ are the Young's modulus and mass density of element $e$ in design $q$, respectively, and $p$ is the exponential power of the SIMP model. The parameters in the SIMP model are set as: $E_{\min }=10^{-9} E_{1}, \quad m_{\min }=10^{-9} m_{1}, \quad p=5$, and Poisson's ratio is $\nu=0.3$.

Table 3. Performance of Different Designs in Fig. 10

\begin{tabular}{lccr}
\hline Design & $f_{v}$ & Band distance $(2 \pi c / a)$ & Bandwidth $(2 \pi c / a)$ \\
\hline Dilated & 0.682 & $8.7 \%$ in $[0.23711,0.25878]$ & 0.00625 in $[0.24473,0.25098]$ \\
Intermediate & 0.611 & $9.9 \%$ in $[0.25299,0.27942]$ & 0.00625 in $[0.26477,0.27102]$ \\
Eroded & 0.537 & $8.3 \%$ in $[0.27770,0.30189]$ & 0.00649 in $[0.28665,0.29314]$ \\
\hline
\end{tabular}




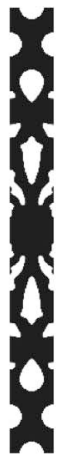

(a)

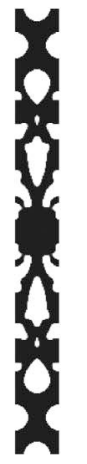

(b)

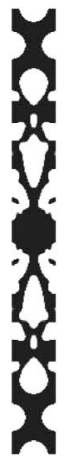

(c)

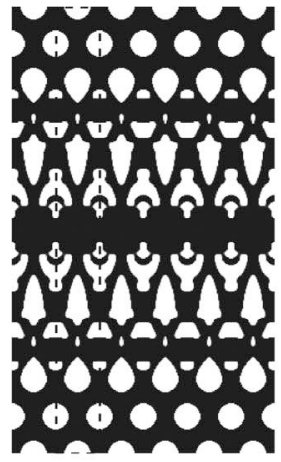

(d)

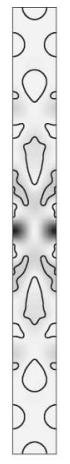

(e)

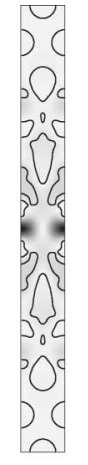

(f)

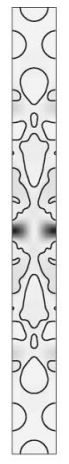

(g)

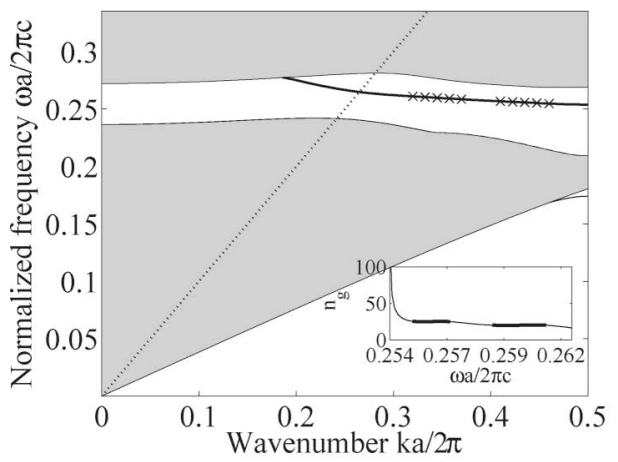

(h)

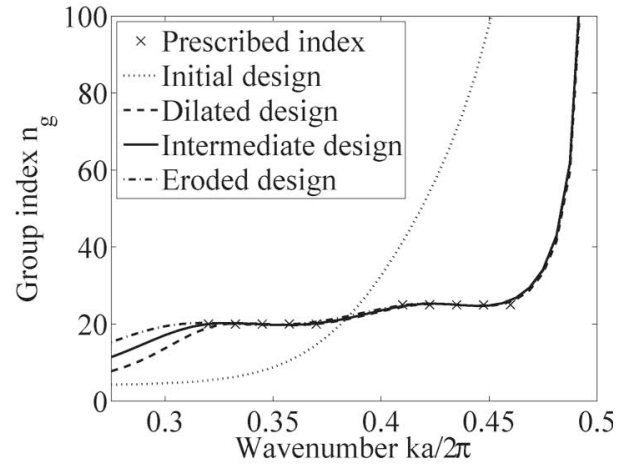

(i)

Fig. 11. Robust design of PhCWs with two constant group indexes. (a) Dilated design. (b) Intermediate design. (c) Eroded design. (d) PhCW constituted by intermediate design. (e) Amplitude of the magnetic field at $k_{i}=0.3452 \pi / a$ for dilated design. (f) Amplitude of the magnetic field at $k_{i}=0.3452 \pi / a$ for intermediate design. (g) Amplitude of the magnetic field at $k_{i}=0.3452 \pi / a$ for eroded design. (h) Band structure of intermediate design (f) Groups indexes of the different design realizations and prescribed group index.

The entire robust formulation can thus be modified as

$$
\begin{gathered}
\min _{\rho_{j}} \max _{q} \max _{k_{i}} f\left(\bar{\rho}^{q}\right)=\left(\frac{c\left(k_{i}-k_{i-1}\right)}{\omega_{n}^{q}\left(k_{i-1}\right)-\omega_{n}^{q}\left(k_{i}\right)}-n_{g}^{*}\right)^{2} \quad \text { s.t. } \quad\left[\mathbf{K}_{k}^{q}-\left(\omega^{q}\right)^{2} \mathbf{M}^{q}\right] \mathbf{h}^{q}=0 \quad\left(\tilde{\mathbf{K}}^{q}-\lambda_{1}^{q} \tilde{\mathbf{M}}^{q}\right) \mathbf{u}^{q}=0 \\
\max _{k_{i i}} \omega_{n-1}^{q}\left(k_{i i}\right) \leq a_{1} \min _{k_{i}} \omega_{n}^{q}\left(k_{i}\right) \quad \omega_{n}^{q}(0) \geq a_{2} \max _{k_{i}} \omega_{n}^{q}\left(k_{i}\right) \quad \min _{k_{i i}} \omega_{n+1}^{q}\left(k_{i i}\right) \geq a_{2} \max _{k_{i}} \omega_{n}^{q}\left(k_{i}\right) \quad \frac{\lambda_{1}^{q}}{\lambda_{0}} \geq \delta, \quad \lambda_{0}=\frac{E_{1}}{m_{1}} \\
f_{v}=\frac{\sum_{j} \bar{\rho}_{j}^{d} v_{j}}{\sum_{j} v_{j}} \leq f_{v}^{*} \quad 0 \leq \rho_{j} \leq 1 \quad j=1, \ldots, N, \quad i=2, \ldots, m, \quad a_{1}<1, \quad a_{2}>1, \quad q=\{d, i, e\} .
\end{gathered}
$$

To illustrate the efficiency of this formulation, we optimize the second example with the same parameter set (shown in Fig. 12) and another example for $n_{g}^{*}=100$ (shown in Fig. 13). The fundamental free vibration frequency constraint is set to $\delta=0.00025$. The normalized bandwidths $\Delta \omega / \omega$ of these two examples are $1.24 \%$ and $0.33 \%$, respec- tively with maximum error of $2 \%$. The final designs show that this new formulation can prevent the isolated components effectively and also performs well for high group indexes. However, it should be noted that the algorithm is quite sensitive to the choice of the parameter $\delta$.

Table 4. Performance of Different Designs in Fig. 11

\begin{tabular}{lcccc}
\hline Design & $f_{v}$ & Band distance $(2 \pi c / a)$ & Bandwidth $(2 \pi c / a)$ of $n_{g}=20$ & Error \\
\hline Dilated & 0.720 & $8.7 \%$ in $[0.22847,0.24939]$ & 0.00250 in $[0.23909,0.24159]$ & $1.7 \%$ \\
Intermediate & 0.648 & $10.5 \%$ in $[0.24210,0.26892]$ & 0.00250 in $[0.25866,0.26116]$ & $1.3 \%$ \\
Eroded & 0.565 & $11.5 \%$ in $[0.26388,0.29623]$ & 0.00284 in $[0.28553,0.28834]$ & $1.6 \%$ \\
\hline
\end{tabular}


Table 5. Performance of Different Designs in Fig. 11

\begin{tabular}{lcc}
\hline Design & Band distance $(2 \pi c / a)$ of $n_{g}=25$ & Error \\
\hline Dilated & 0.00175 in $[0.23741,0.23566]$ & $1.2 \%$ \\
Intermediate & 0.00175 in $[0.25495,0.25670]$ & $1.7 \%$ \\
Eroded & 0.00200 in $[0.28355,0.28155]$ & $1.8 \%$ \\
\hline
\end{tabular}

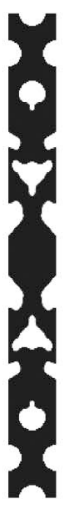

(a)
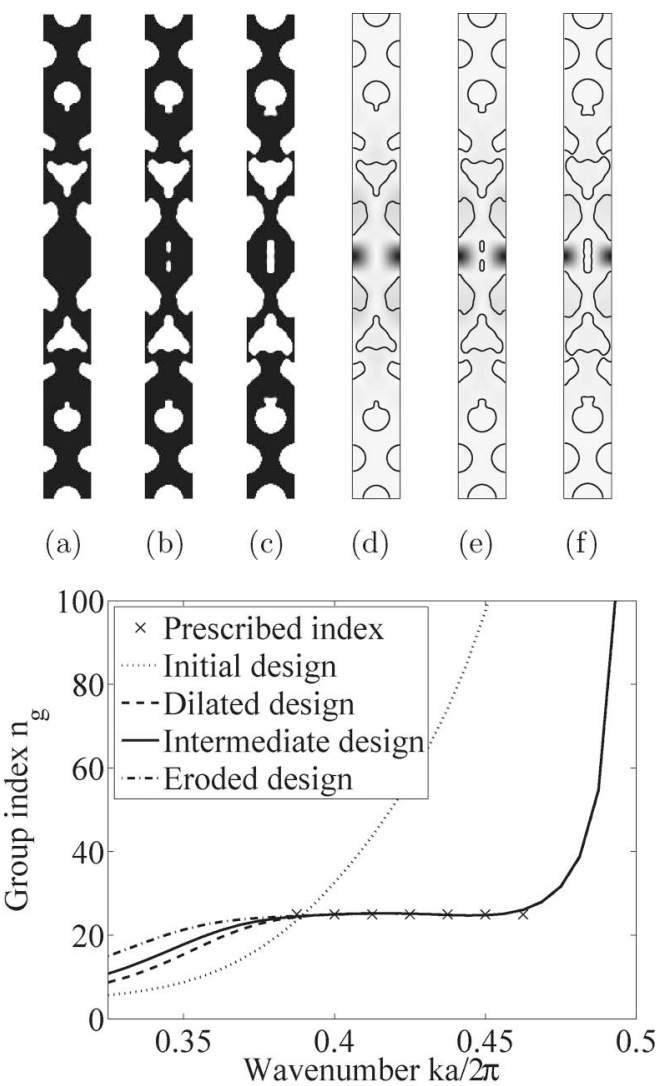

(g)

Fig. 12. Robust design of PhCWs with fundamental free vibration frequency constraint. (a) Dilated design. (b) Intermediate design. (c) Eroded design. (d) Amplitude of the magnetic field at $k_{i}=$ $0.4252 \pi / a$ for dilated design. (e) Amplitude of the magnetic field at $k_{i}=0.4252 \pi / a$ for intermediate design. (f) Amplitude of the magnetic field at $k_{i}=0.4252 \pi / a$ for eroded design. (g) Group indexes of the different design realizations and prescribed group index.

\section{CONCLUSION}

In this study, a robust topology optimization method to tailor the dispersion properties of PhCWs has been presented. The objective is formulated as a min-max optimization problem based on the errors between actual group indexes and a prescribed group index among the dilated, intermediate, and eroded designs, which mimic under, normal, and overetching in the manufacturing process. Band constraints are implemented to isolate the design modes from other modes. This formulation ensures that if the proposed design is slightly dilated or eroded during manufacturing, the PhCW still preserves the desired performance. The proposed robust formulation indirectly ensures a minimum length scale on important design features (the response of the optimized design is tolerant towards errors in the realization of smaller details). The minimum length scale can be controlled by proper selection of

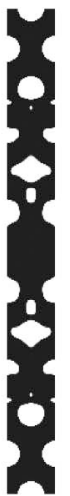

(a)

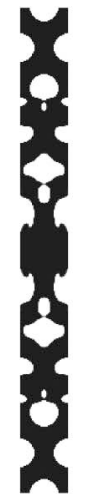

(b)

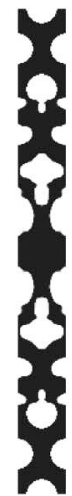

(c)

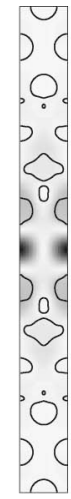

(d)

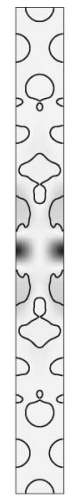

(e)

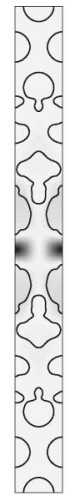

(f)

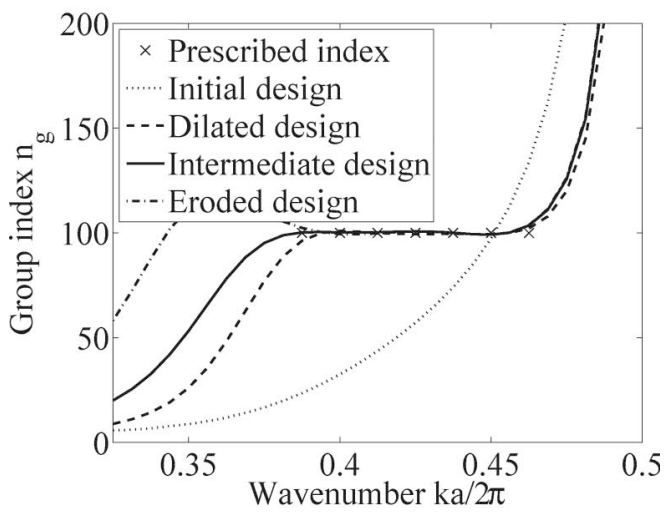

(g)

Fig. 13. Robust design of PhCWs with $n_{g}=100$. (a) Dilated design. (b) Intermediate design. (c) Eroded design. (d) Amplitude of the magnetic field at $k_{i}=0.4252 \pi / a$ for dilated design. (e) Amplitude of the magnetic field at $k_{i}=0.4252 \pi / a$ for intermediate design. (f) Amplitude of the magnetic field at $k_{i}=0.4252 \pi / a$ for eroded design. (g) Group indexes of the different design realizations and prescribed group index.

filter size and projection values (see [24] for more details). PhCWs with group index of $n_{g}=25$ are demonstrated with very small GVD and the bandwidth of slow light of $n_{g}=25$ can be increased to $0.006252 \pi c / a$ through the robust design. The detailed properties of the PhCWs reveal that the reduction of the GVD and the extension of the bandwidth are at expense of the relative band distance. Another example shows that a slow-light PhCW with $n_{g}=100$ can be achieved with a bandwidth of $0.000792 \pi c / a$. Moreover, PhCWs with novel functionality, exhibiting two constant group index regions over different frequency ranges, can be created by the robust design formulation. The appearance of isolated components in the overetching are avoided by introducing a fundamental free mechanical vibration constraint. The proposed design method provides a systematic and robust method for tailoring the dispersion of PhCWs.

\section{ACKNOWLEDGMENTS}

This work was financially supported by Villum Fonden (via the NATEC Centre of Excellence), the Eurohorcs/ESF European Young Investigator Award (EURYI, www.esf.org/ euryi), and the Danish Center for Scientific Computing (DCSC). 


\section{REFERENCES}

1. T. Baba, "Slow light in photonic crystals," Nat. Photon. 2 465-473 (2008).

2. J. D. Joannopoulos, S. G. Johnson, J. N. Winn, and R. D. Meade, Photonic Crystals-Molding the Flow of Light, 2nd ed. (Princeton University Press, 2008).

3. D. Mori and T. Baba, "Wideband and low dispersion slow light by chirped photonic crystal coupled waveguide," Opt. Express 13, 9398-9408 (2005).

4. D. Mori, S. Kubo, H. Sasaki, and T. Baba, "Experimental demonstration of wideband dispersion-compensated slow light by a chirped photonic crystal directional coupler," Opt. Express 15, 5264-5270 (2007).

5. L. H. Frandsen, A. V. Lavrinenko, J. Fage-Pedersen, and P. I. Borel, "Photonic crystal waveguides with semi-slow light and tailored dispersion properties," Opt. Express 14, 9444-9450 (2006).

6. J. Li, T. P. White, L. O'Faolain, A. Gomez-Iglesias, and T. F. Kraus, "Systematic design of flat band slow light in photonic crystal waveguides," Opt. Express 16, 6227-6232 (2008).

7. S. Kubo, D. Mori, and T. Baba, "Low-group-velocity and lowdispersion slow light in photonic crystal waveguides," Opt. Lett. 32, 2981-2983 (2007).

8. M. P. Bendsøe and O. Sigmund, Topology OptimizationTheory, Methods and Applications (Springer Verlag, 2004).

9. P. I. Borel, A. Harpøth, L. H. Frandsen, M. Kristensen, J. S. Jensen, P. Shi, and O. Sigmund, "Topology optimization and fabrication of photonic crystal structures," Opt. Express 12, 1996-2001 (2004).

10. J. S. Jensen and O. Sigmund, "Systematic design of photonic crystal structures using topology optimization: low-loss waveguide bends," Appl. Phys. Lett. 84, 2022-2024 (2004).

11. J. S. Jensen and O. Sigmund, "Topology optimization of photonic crystal structures: a high-bandwidth low-loss $T$-junction waveguide," J. Opt. Soc. Am. B 22, 1191-1198 (2005).

12. J. S. Jensen and O. Sigmund, "Topology optimization for nanophotonics-a review," Laser Photon. Rev. (to be published).

13. R. Stainko and O. Sigmund, "Tailoring dispersion properties of photonic crystal waveguides by topology optimization," Waves in Random and Complex Media 17, 477-489 (2007).
14. O. Sigmund, "On the design of compliant mechanisms using topology optimization,” Mechan. Struct. Mach. 25, 493-524 (1997).

15. O. Sigmund and J. Petersson, "Numerical instabilities in topology optimization: a survey on procedures dealing with checkerboards, mesh-dependencies and local minima," Struct. Opt. 16, 68-75 (1998).

16. T. E. Bruns and D. A. Tortorelli, "Topology optimization of nonlinear elastic structures and compliant mechanisms," Comput. Methods Appl. Mech. Eng. 190, 3443-3459 (2001).

17. B. Bourdin, "Filters in topology optimization," Int. J. Numer Methods Eng. 50, 2143-2158 (2001)

18. J. K. Guest, J. H. Prevost, and T. Belytschko, "Achieving minimum length scale in topology optimization using nodal design variables and projection functions," Int. J. Numer. Methods Eng. 61, 238-254 (2004).

19. M. Y. Wang and S. Wang, "Bilateral filtering for structural topology optimization," Int. J. Numer. Methods Eng. 63, 1911-1938 (2005).

20. O. Sigmund, "Morphology-based black and white filters," Struct Multidisc. Optim. 33, 401-424 (2007).

21. J. K. Guest, "Topology optimization with multiple phase projection," Comput. Methods Appl. Mech. Eng. 199, 123-135 (2009).

22. S. Xu, Y. Cai, and G. Cheng, "Volume preserving nonlinear density filter based on heaviside functions," Struct. Multidisc. Optim. 41, 495-505 (2009).

23. O. Sigmund, "Manufacturing tolerant topology optimization," Acta Mech. Sin. 25, 227-239 (2009).

24. F. Wang, B. S. Lazarov, and O. Sigmund, "On projection methods, convergence and robust formulations in topology optimization," Struct. Multidisc. Optim. (to be published).

25. A. P. Seyranian, E. Lund, and N. Olhoff, "Multiple eigenvalues in structural optimization problems," Struct. Multidisc. Optim. 8, 207-227 (1994)

26. N. L. Pedersen and A. K. Nielsen, "Optimization of practical trusses with constraints on eigenfrequencies, displacements," Struct. Multidisc. Optim. 25, 436-445 (2003).

27. K. Svanberg, "A class of globally convergent optimization methods based on conservative convex separable approximations," SIAM J. Optim. 12, 555-573 (2002). 